\title{
Comparison of Alvarado Score and Paediatric Appendicitis Score for Diagnosing Appendicitis in Children
}

\section{Naeem Liaqat, ${ }^{1}$ Asif Iqbal, ${ }^{2}$ Wajeeh Ur Reham, ${ }^{3}$ Zulfiqar Ahmed, ${ }^{4}$ Fozia Bashir, ${ }^{5}$ Sajid Hameed Dar ${ }^{6}$}

\begin{abstract}
Objective: To compare diagnostic accuracy of Alvarado score (AS) and Paediatric Appendicitis Score (PAS) for diagnosis of acute appendicitis in children.

Methods: This study was conducted at the department of Pediatric Surgery Children Hospital Lahore, over a period of 1 year. All the patients undergoing appendicectomy were included. Alvarado score and Pediatric Appendicitis score (PAS) was evaluated, compared and appendix specimen sent for histopa-thology. All findings were recorded in proforma. The collected data was analyzed by SPSS version 24 . The mean Alvarado score and PAS was calculated, and stratified according to the histopathology reports. The sensitivity and specificity of both Alvarado score and PAS for three strata including score 3-5, 5-7 and 8-10 were also calculated.

Results: A total of 177 patients were included in the study. The mean age of the patients was $9.16 \pm 2.386$ years. Among these 118 patients (67\%) were male. The mean duration of pain was $21.42 \pm 19.05$ hours. Biopsy report showed that 18 patients $(10.1 \%)$ had normal appendix with no signs of inflammations while 159 patients $(89.9 \%)$ had inflammation on histopathology. We stratified the histopathology reports according to Alvarado score $\leq 7$ and $>7$ and P-vale was found significant. Similarly PAS $\leq 7$ and $>7$ was stratified and Pvalue was not significant. The difference in mean Alvarado score between having acute appendicitis and those with normal histopathology was significant $(\mathrm{P}=0.000)$ while this difference in mean PAS was not found significant $(\mathrm{P}=0.325)$.

Conclusions: None of the scoring system has adequate diagnostic accuracy and clinical judgment is preferred.

Key Words: Alvarado Score; PAS; Appendicitis; Children

How to cite: : Liaqat N., Iqbal A., Rehman ur W., Ahmed Z., Bashir F., Dar H.S. Comparison of Alvarado score and Paediatric Appendicitis Score for diagnosing appendicitis in children" Esculapio 202 1;17(02):176-179.

DOI: https://doi.org/10.51273/esc21.2517213
\end{abstract}

\section{Introduction}

A cute appendicitis (AS) is the most common pediatric surgical emergency and appendectomy is the most commonly performed procedure in children. ${ }^{1}$ AA is the inflammation if appendix with classical pre-

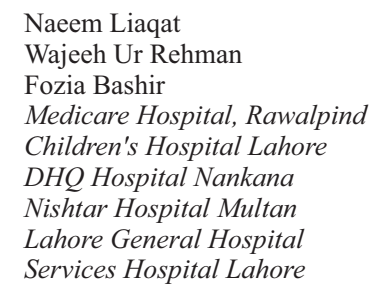

Naeem Liaqat

Wajeeh Ur Rehman

Fozia Bashir

Medicare Hospital, Rawalpind

Children's Hospital Lahore

DHQ Hospital Nankana

Nishtar Hospital Multan

Lahore General Hospital

Services Hospital Lahore

Correspondence:

Dr. Naeem Liaqat, Assistant Professor, Medicare Hospital, Rawalpind Email: isimsonian.chaudary@gmail.com

Submission Date:

1st Revision Date:

Acceptance Date:
2. Asif Iqbal

4. Ahmed

6. Sajid Hameed Dar sentation in the form of anorexia, nausea or vomiting, fever, periumbilical pain initially which may later shift to right iliac fossa. ${ }^{2}$ On examination, there is tachycardia, tenderness, and rebound tenderness. However, the signs and symptoms may vary and the clinical diagnosis is tricky especially in children who cannot localize pain. There are no other diseases that have such a variety of symptoms as AA. Also, the classical signs and symptoms are mostly absent in $20-33 \%$ of the patients and due to this many children present late with complications like perorated appendix, abscess or peritonitis. $^{3,4}$

Delayed or missed diagnosis have the potential to result in significant morbidity from appendiceal perforation, abscess formation, wound infection, wound dehiscence and even mortality. However, negative diagnosis of 
acute appendicitis exposes children to unnecessary operation. ${ }^{5}$

Many scoring systems were devised for the diagnosis of acute appendicitis including Alvardo score (AS), RIPASA, Samuel, Pediatric Appendicitis Score (PAS) which can help the physician and the surgeon to finally reach a diagnosis. AA is quite a rare in neonatal period of life and is much common in childhood and early adult life. ${ }^{6}$ Generally, these clinical scoring systems are more informative than specific symptoms or signs alone. Still, they are not capable of predicting appendicitis with sufficient probability and therefore should not be used alone to diagnose it. ${ }^{7}$ There is still a debate regarding the diagnostic accuracy of these scoring systems for AA. ${ }^{8}$ However, most commonly used scores are AS and PAS. No specific data or study is available which compares the diagnostic accuracy of these scores so it is difficult to say which scores is helpful in making the diagnosis. We conducted this study to compare the diagnostic accuracy ofAS and PAS.

\section{Methods}

We conducted this prospective study at The Department of Pediatric Surgery of The Children's Hospital \& the Institute of child health, Lahore. The study took place over a period of 1 year from January 2018 till December 2018. Ethical approval was taken before the start of study. All the pediatric patients undergoing appendicectomy were included in the study. A pre designed proforma was used for data collection which consist of four parts, first part include the demographic details of patients, second part had two scoring systems i.e. (AS and PAS). While the $3^{\text {rd }}$ and $4^{\text {th }}$ part of proforma were about the operative and histopathology findings. In all patients AS and PAS were evaluated and findings were recorded on proforma. The patients included in the study underwent appendectomies appendicular tissue was sent for histopathology. Sampling was done by the method of consecutive sampling.

The collected data was analyzed by SPSS version 24 . The mean of Alvarado score and PAS was calculated, we also stratified the histopathology reports to Alvarado score and PAS. The sensitivity and specificity of both Alvarado score and PAS for three strata including score 3-5, 5-7 and 8-10 were also calculated.

\section{Results}

A total of 177 patients were included in the study. The mean age of the patients was found to be $9.16 \pm 2.386$ years. Among these 118 patients (67\%) were male while remaining 59 patients $(33 \%)$ were females. The mean duration of pain was $21.42 \pm 19.05$ hours. Seventy six percent of patients $(n=135)$ were having history of pain for less than 24 hours while 42 patients (24\%) had history of more than 24 hours. Per-operative findings of all these patients are summarized in figure 1. Biopsy report showed that 18 patients $(10.1 \%)$ had normal appendix with no signs of inflammations while 159 patients $(89.9 \%)$ had inflammation on histopathology. It also showed that 41 patients who had normal looking appendix ultimately showed signs of inflammation in 23 specimens. We stratified the histopathology reports according to Alvarado score 7 and $>7$ and P-vale was found significant. Similarly PAS 7 and $>7$ was stratified and P-value found not significant (Table1). The difference in mean Alvarado score between having acute appendicitis and those with normal histo-pathology was significant $(\mathrm{P}=0.000)$ while this diffe-rence in mean PAS was not found significant $(\mathrm{P}=0.325)$ (Table 2$)$. The sensitivity and specificity of both Alva-rado score and PAS for three strata including score 3-5, 5-7 and 8-10 were calculated and summarized in Table 3 and 4.

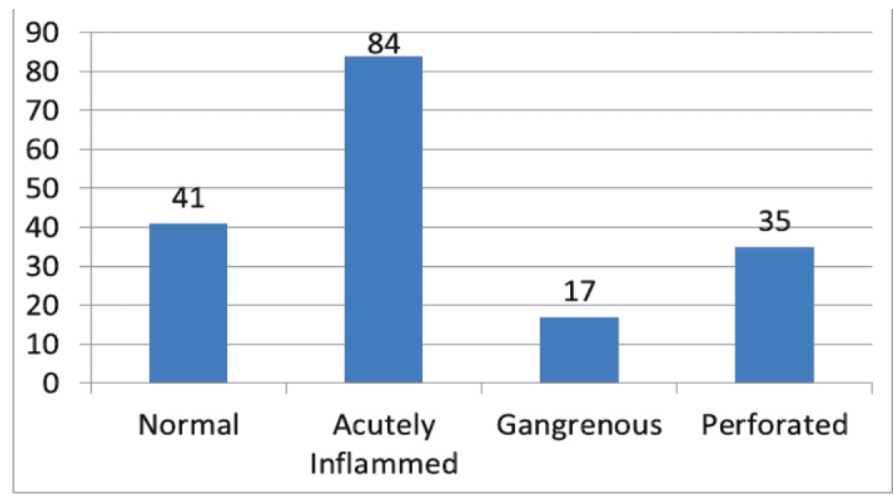

Figure 1: Intra Operative Findings

Table 1: Stratification of histopathology with Alvarado score and PAS

\begin{tabular}{lcc}
\hline & Normal & $\begin{array}{c}\text { Acutely } \\
\text { Inflammed }\end{array}$ \\
\hline Alvarado Score upto 7 & 15 & 68 \\
Alvarado Score more than 7 & 3 & 91 \\
PAS upto 7 & P Value $=\mathbf{0 . 0 0 1}$ & \\
PAS more than 7 & 6 & 55 \\
& 12 & 104 \\
\hline
\end{tabular}


Table 2: Mean Alvarado Score and PAS in Cases having Acute Appendicitis

\begin{tabular}{lccc}
\hline & Normal & $\begin{array}{c}\text { Acutely } \\
\text { Inflammed }\end{array}$ & $\begin{array}{c}\text { P- } \\
\text { Value }\end{array}$ \\
\hline Alvarado Score (mean) & 5.277 & 7.37 & 0.000 \\
Pediatric Appendicitis Score & 7.277 & 7.68 & 0.325 \\
(mean) & & & \\
\hline
\end{tabular}

Table 3: The sensitivity and Specificity of Alvarado Score \& PAS for Three Strata Including: Score 3-5, 5-7 and 8-10

\begin{tabular}{llccc}
\hline & & $\mathbf{3 - 5}$ & $\mathbf{6 - 7}$ & $\mathbf{8 - 1 0}$ \\
\hline \multirow{2}{*}{ Alvarado } & Specificity & $23.9 \%$ & $18.8 \%$ & $57.2 \%$ \\
& Sensitivity & $38.8 \%$ & $77.7 \%$ & $83.3 \%$ \\
\multirow{3}{*}{ PAS } & Specificity & $8.8 \%$ & $25.7 \%$ & $65.4 \%$ \\
& Sensitivity & $83.33 \%$ & $83.33 \%$ & $33.3 \%$ \\
\hline
\end{tabular}

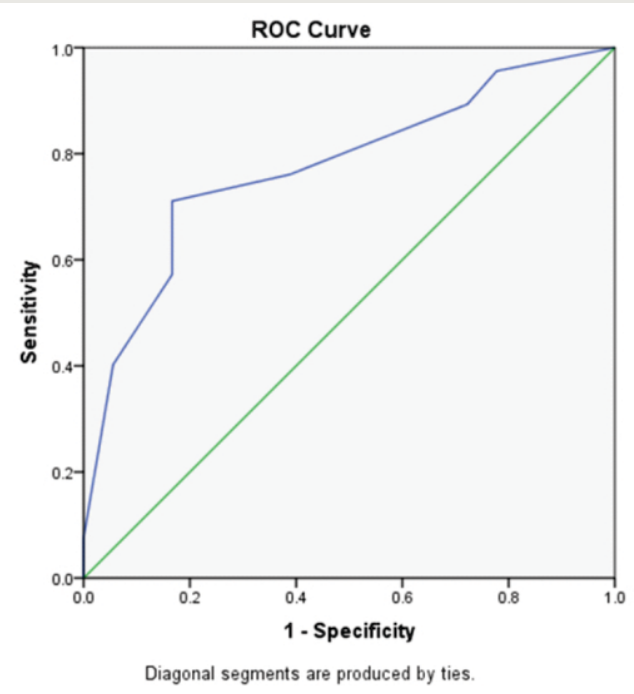

Figure-2: Receiver Operator Characteristic Curves: Alvarado

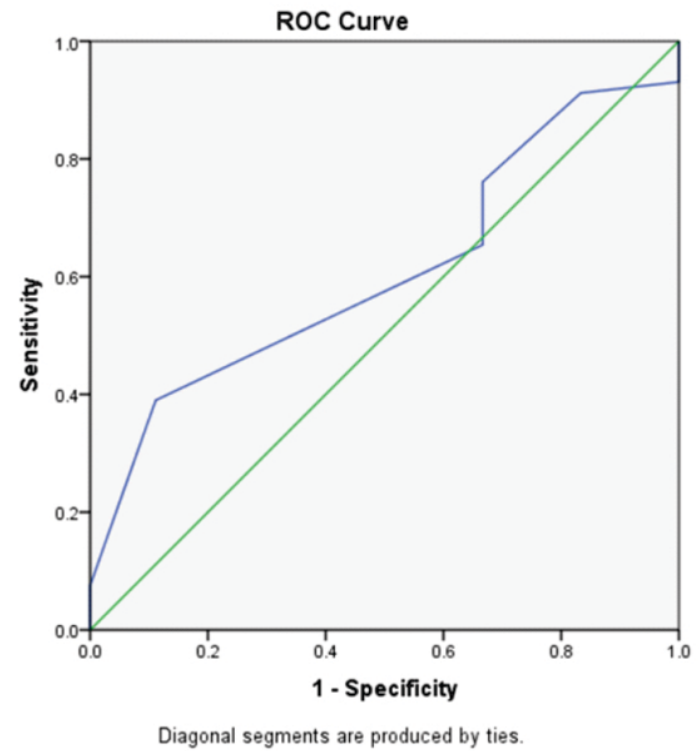

Figure-3: Receiver Operator Characteristic Curves: PAS

\section{Discussion}

AA is one of the most common surgical diseases seen in surgical emergency however it can be often challenging for surgeon to make a correct diagnosis in order to reduce the chance of negative exploration for acute appendicitis. ${ }^{9}$ It very important to differentiate pain abdomen secondary to inflammation of appendix from other causes, which is often a difficult task especially in children. ${ }^{10}$ Early and accurate diagnosis reduces the rate of post-operative morbidity \& mortality. The diagnosis of appendicitis is mainly clinical with help from radiology and laboratory may be needed for confirmation. Over time many scoring systems had been developed and Alvarado score is one of the most widely used tool for the diagnosis of appendicitis in adults but it is showed varied results in children. It consists of eight clinical and laboratory assessment items. ${ }^{11}$ In 2002 PAS was developed by Samuel for children between 4 to 15 years of age it is a modification of Alvarado Score and carries a maximum score of 10.8 In this study we tried to compare a well reputed Alvarado scoring system with pediatric appendicitis score with regard to correct diagnosis based on operative findings.

We found out the mean age of the patients was $9.16 \pm$ 2.386 years. It was comparable with Samuel who reported in cohort for development of PAS. ${ }^{12}$ In current study the difference in mean Alvarado score between having acute appendicitis and those with normal histopathology was significant $(\mathrm{P}<0.001)$, while this difference in mean PAS was not found significant ( $p-0.57$ ). But a similar study showed significant $\mathrm{P}$-Value $(\mathrm{P}<$ 0.001 ) for both Alvardo \& PAS in relation to appendicitis versus normal appendix. ${ }^{13}$

In present study, the sensitivity and specificity of both Alvarado score and PAS for three strata including score $3-5,5-7$ and $8-10$ were $23.9 \%, 18.8 \%, 57.2 \%$ and $8.8 \%, 25.7 \%, 65.4 \%$ respectively. Pogorelic et al reported that in 236 patients (sensitivity, 89\%; specificity, $59 \%$; positive predictive value, $93.1 \%$ ), whereas in patients with acute appendicitis and a PAS of 7 or higher, the correct diagnosis would have been set in 228 patients (sensitivity, $86 \%$; specificity, $50 \%$; positive predictive value, $90.1 \%$ ). No significant difference was found in sensitivity and specificity between the observed scoring systems they suggested that these scores can only provide assistance. ${ }^{14}$ However a study by Kim in adults showed, the sensitivity of the Alvarado score $86.2 \%$, its specificity $61.6 \%$, and the accuracy 
of diagnosis was $82.9 \% .{ }^{15}$ Another study publishd in an Indian journal reported that The sensitivity of PAS was 0.87 , specificity 0.59 , positive predictive value 0.83 , and negative predictive value 0.67 . This study concluded that the PAS score had $17 \%$ negative appendicectomy rate of and an unacceptable $13 \%$ of patients with appendicitis being missed. ${ }^{16}$

Our results are comparable to Badebarin D et al., who reported that although the diagnostic value of Alvarado score is higher as compared to PAS but the sensitivity, specificity, PPV , NPV are not satisfactory for conformation of diagnosis. ${ }^{17}$

\section{Conclusion}

Both the scores failed to show the desired sensitivity and specificity and therefore it was concluded that clinical assessment should be done for making the final diagnosis.

\section{Conflict of Interest: None}

\section{References}

1. Almaramhy HH. Acute appendicitis in young children less than 5 years: review article. Ital J Pediatr. 2017 Jan 26;43(1):15. doi: 10.1186/s13052-017-0335-2. PMID: 28257658; PMCID: PMC5347837.

2. Dogra BB. Acute appendicitis: Common surgical emergency. Medical Journal of Dr. DY Patil University. 2014 Nov 1;7(6):749

3. Gadiparthi R, Waseem M. Pediatric Appendicitis. [Updated 2019 Dec 16]. In: StatPearls [Internet]. Treasure Island (FL): StatPearls Publishing; 2020 Jan-. Available from:

4. Kim D, Butterworth SA, Goldman RD. Chronic appendicitis in children. Can Fam Physician. 2016 Jun; 62 (6): e304-5. PMID: 27303020; PMCID: PMC4907572.

5. Jones K, Pena AA, Dunn EL, et al. Are negative appendectomies still acceptable? Am J Surg 2004; 188: $748 \mathrm{e} 754$.

6. Naoum JJ, Mileski WJ, Daller JA, et al. The use of abdominal computed tomography scan decreases the frequency of misdiagnosis in cases of suspected acute appendicitis. Am J Surg. 2002;184:587-589.

7. Brown TW, McCarthy ML, Kelen GD, et al. An epidemiologic study of closed emergency department malpractice claims in a national database of physician malpractice insurers. Acad Emerg Med.2010; 17(5): 553--560.

8. Bergeron E. Clinical judgment remains of great value in the diagnosis of acute appendicitis. Can J
Surg. 2006; 49(2):96--100.

9. Derakhshanfar H, Pourbakhtyaran E, Rahimi S, Sayyah S, Karbasian F, Soltantooyeh Z, Fallah S. Survey of efficacy of pediatric appendicitis score in Iranian patients less than 18 years old referred to the emergency department. J Family Med Prim Care. 2019 Nov 15; 8(11):3700-3705. doi: 10.4103/jfmpc.jfmpc_740_19. PMID: 31803676; PMCID: PMC6881965.

10. Kalan M, Talbot D, Cunliffe WJ, et al. Evaluation of the modified Alvarado score in the diagnosis of acute appendicitis: a prospective study. Ann R Coll Surg. 1994;76:418-419

11. Pogorelic Z, Rak S, Mrklic I, Juric I. Prospective validation of Alvarado score and Pediatric Appendicitis Score for the diagnosis of acute appendicitis in children. Pediatric emergency care. 2015 Mar 1;31(3):164-8.

12. Goulder F, Simpson T. Pediatric appendicitis score: A retrospective analysis. J Indian Assoc Pediatr Surg. 2008;13(4):125-127. doi:10.4103/0971-9261.44761

13. Parveen KZ, Avabratha KS, Shetty K. Pediatric appendicitis score in the diagnosis of childhood appendicitis: a validation study. Int J Contemp Pediatr. 2017 Nov; 4(6): 2196.

14. Teixeira PG, Sivrikoz E, Inaba K, Talving P, Lam L, Demetriades D. Appendectomy timing: waiting until the next morning increases the risk of surgical site infections. Annals of surgery. 2012 Sep 1;256(3):53843.

15. Chung PH, Dai K, Yang Z, Wong KK. Validity of Alvarado Score in predicting disease severity and postoperative complication in pediatric acute appendicitis. World Journal of Pediatric Surgery. 2019 Feb 1;2(1).

16. Rajbhandari B, Dahal GR, Pokharel RP. Comparison Between Alvarado Score and Paediatric Appendicitis Score in Diagnosing Acute Appendicitis in Children. Journal of Nepal Paediatric Society. 2020 Dec 15; 40(3):178-84.

17. Badebarin, D., Parsay, S., \& Aslanabadi, S. (2020). Alvarado vs. Pediatric Appendicitis Score (PAS) in Acute Appendicitis of Children. Iranian Journal of Pediatric Surgery, 6(1), 1-9. https:// doi.org/ 10.22037/ irjps.v6i1.31696

\section{Authors Contribution}

N.L: Conceptualization of Project

A.I,Z.A: Data Collection

W.Ur.R: Literature Search

F.B: Drafting, Revision

S.H.D: Literature Search 